\title{
¿Quiénes se benefician del turismo en Cayos Cochinos, Honduras?
}

\author{
Sebastian Kuch* \\ Universidad Nacional \\ Autónoma de México
}

\section{Resumen}

El Monumento Natural Marino Archipiélago Cayos Cochinos (mNмcc) es un área protegida en el mar Caribe frente a la costa norte de Honduras. La Fundación Hondureña para la Protección y Conservación de los Cayos Cochinos (HCRF) es una organización no gubernamental que lidera el manejo común del área y fomenta el desarrollo turístico de uso sostenible y no consuntivo de los recursos naturales y marinos de la zona, es decir, como una actividad económica alternativa para las comunidades garífunas locales pesqueras. Debido a la ausencia del Estado en cuanto a la conducción y financiamiento de áreas protegidas, la HCRF ha establecido asociaciones con el sector privado vinculado a la industria turística, con la finalidad de generar fondos para llevar a cabo sus programas de conservación. Sin embargo, esto ha generado una creciente interdependencia de los objetivos de conservación ambiental y cultural estipulados para el MNMCC con la operabilidad financiera de la HCRF y con los fines de lucro de las empresas privadas. Estas asociaciones influyen en gran medida sobre los derechos ancestrales y los intereses de las comunidades garífunas, manipulando las relaciones de ellas con la HCRF.

\section{Palabras clave}

Reality show, garífunas, Fundación Hondureña para la Protección y Conservación de los Cayos Cochinos (HCRF), Monumento Natural Marino Archipiélago Cayos Cochinos (MNMCC), turismo.

Recibido: 11/04/2014 · Aceptado: 26/06/2014

*Correo electrónico: sebastiankuch@gmail.com 


\title{
Who benefits form tourism in Cayos \\ Cochinos, Honduras?
}

\author{
Sebastian Kuch* \\ Universidad Nacional \\ Autónoma de México
}

\begin{abstract}
The Natural Marine Monument Archipiélago Cayos Cochinos (MNMcc) is a protected area in the Caribbean on the northern Honduras coastline. The Honduran Foundation for the protection and conservation of Cayos Cochinos (HCRF) is a NGo that leads the common use of the area supporting sustainable tourism development and non-consumptive use of natural and marine i.e. an alternative economic activity for the local garifuna communities. Because of the lack of funding and guidance by the Government, HCRF has established alliances with the private hotel industry to obtain funds for their conservation projects. By doing so, certain interdependent conflicts have been generated between the cultural and environmental conservation objectives of MNMcc, those related to financial operation of HCRF and those related to profit within private industry. These alliances have an influence upon the ancestral rights and interests of garifuna communities manipulating their relations with HCRF.
\end{abstract}

\section{KEY WORDS}

Garífunas, Fundación Hondureña para la Protección y Conservación de los Cayos Cochinos (HCRF), Monumento Natural Marino Archipiélago Cayos Cochinos (MNMCC), tourism.

*E-mail: sebastiankuch@gmail.com 


\section{Introducción}

El archipiélago Cayos Cochinos es un grupo de quince islas en el mar Caribe frente a la costa norte de Honduras. La mayoría de estos cayos se encuentra en posesión privada de empresarios hondureños y extranjeros. Tradicionalmente, los caladeros de esta área ecológica sensible han sido importantes medios de subsistencia para las comunidades garífunas de la zona. Una vez que el archipiélago fue declarado área protegida en 1993, se otorgó el manejo a organizaciones conservacionistas privadas, primero a la Smithsonian Institution (19931997) y después a la Fundación Hondureña para la Protección y Conservación de los Cayos Cochinos (en inglés Honduran Coral Reef Foundation, HCRF). En este periodo se han establecido e impuesto amplias restricciones de pesca a las comunidades locales, lo cual afecta sobremanera sus rutinas habituales de trabajo. Para reducir la dependencia de la pesca, la HCRF fomenta el desarrollo turístico sostenible en el archipiélago, como una actividad económica alternativa para la población (Bown, 2010: 4-6; Denning, 2005: 139-141; Courrau y Andraka, 2004: 13; HCRF, 2004: 24 y 46 y 2008: 81).

En los últimos años, el turismo se ha convertido en un factor clave dentro de la política financiera de la HCRF. Sin embargo, pese a que la actividad turística ha garantizado su propio funcionamiento operativo no ha impulsado el desarrollo socioeconómico y cultural de los habitantes de la zona. En efecto, la HCRF ha establecido asociaciones con empresas privadas, lo que ha resultado en el impulso y la expansión de un turismo que no necesariamente está en concordancia con la política conservacionista, los intereses comunitarios y la capacidad de carga del área protegida.

El presente estudio mostrará que:

a) La HCRF promueve el desarrollo turístico en el Monumento Natural Marino Archipiélago Cayos Cochinos (MNMcc) a través de la empresa privada para garantizar su propia operación financiera, siendo mínimos los beneficios tangibles para las comunidades garífunas.

b) Esta política difiere del objetivo primordial de implantar el turismo en el área como un modelo socioeconómico alternativo para la población local.

c) El actual desarrollo turístico contradice tanto las reivindicaciones de un turismo sostenible postuladas por la HCRF como los objetivos de conservación 
e integridad étnica-cultural de las comunidades garífunas del MNMcc, culminando en una política de doble rasero, que privilegia los intereses privados y discrimina a las comunidades.

\section{Justificación}

El estudio tiene su justificación central en la creciente importancia del turismo en el MNMcc, en particular como:

- una actividad desarrollada por la empresa privada,

- una fuente de ingresos para la HCRF, a fin de sostener los proyectos de conservación, y

- una actividad socioeconómica no tradicional en las comunidades garífunas del archipiélago.

Los atractivos turísticos más llamativos del MNMCc se relacionan con el turismo de sol y playa. La mayoría de los flujos de visitantes corresponde a excursionistas que visitan de día el archipiélago, en un tour organizado (Pineda y Aronne, 2012: 7). No obstante, cuantitativa y cualitativamente, son relevantes otros tipos de turismo. En la zona se practican sobre todo tres clases de turismo: el de crucero, el voluntario-científico y la filmación de reality shows.

El principal atractivo turístico de la zona es el archipiélago de los Cayos Cochinos (con una superficie de $485337 \mathrm{~km}^{2}$ ), pues estos están rodeados de arrecifes coralinos, playas, manglares y aguas cristalinas (HCRF, 2008: 14). Es por ello que, tras la declaratoria del área protegida en 1993, la HCRF propuso y trabajó para que en 2003 se le otorgara el status de Monumento Natural Marino. A la importancia ecológica en el mNMcc debe agregarse que ahí se encuentran las dos comunidades garífunas insulares con menor población del país, estas residen en el pequeño cayo de Chachahuate y en Cayo Cochino Menor, donde se ubica la comunidad de East End. Las dos comunidades mantienen vínculos familiares y comerciales con los pueblos garífunas costeros de Sambo Creek, Nueva Armenia y Río Esteban (mapa 1). 


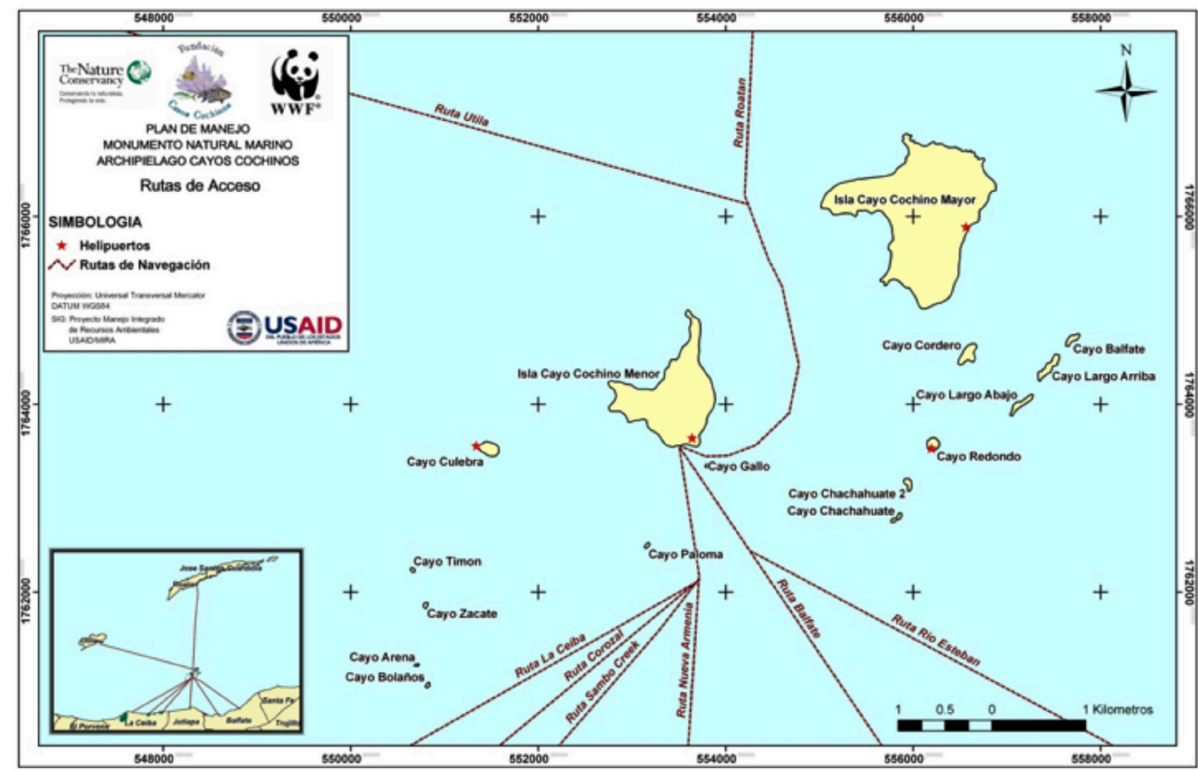

Fuente: HCRF, 2008.

Mapa 1. Monumento Nacional Marino Archipiélago Cayos Cochinos

Los caladeros del MNMcc han sido medios de subsistencia de mucha trascendencia para los pescadores garífunas del área. Antes de que el archipiélago fuera declarado área protegida, grupos de pescadores garífunas se habían asentado de manera permanente en los Cayos Cochinos y hacían uso de sus recursos marinos (Brondo y Bown, 2011: 94). Con el paso del tiempo, y con la finalidad de reducir la dependencia de estas comunidades respecto de la pesca, la HCRF -apoyada por otras organizaciones no gubernamentales, onG- introdujo el turismo como un nuevo modelo para el desarrollo socioeconómico comunitario (Brondo y Woods, 2007: 2).

En todo el mundo se acepta que en muchas áreas protegidas se desarrollan actividades turísticas. La compatibilidad de la conservación con la actividad turística se basa en la presunción de que el turismo no solo representaría un uso menos consuntivo y más sostenible de los recursos naturales y/o culturales en comparación con otras actividades económicas, y de que a la vez generaría fondos para la creación y el mantenimiento de las áreas protegidas (Denning, 2005: 
12-14; Moreno, 2005: 218-219; Meethan, 2002: 60-61). Esto se aplica en particular a países en vías de desarrollo, donde el Estado no dispone de los recursos financieros y humanos para garantizar la conservación de la biodiversidad y los testimonios culturales (Aderhold, 2000: 37). Este turismo, concebido como una opción diferente del de masas, conocido como turismo alternativo, tiene distintas denominaciones: eco-, etno- o geoturismo, que por lo general acentúan el uso sostenible de los recursos naturales y culturales, con el propósito de tener un impacto positivo sobre la economía local (Friedl, 2002: 50-51).

En el caso del MNMCc, la HCRF defiende y promueve un turismo "sin dejar rastros", el cual implica que: "la presencia de turistas en un área no sea evidente; es decir, que no dejen rastros y que, al marcharse, los sitios visitados queden iguales o mejor que antes de la visita” (Courrau y Andraka, 2004: 14).

En estricto sentido, la reivindicación de un turismo sostenible no debe tener en cuenta únicamente los aspectos de la conservación ambiental sino también los derechos y la integridad étnica-cultural de las comunidades turísticas -en este caso, garífunas- del sitio, así como el desarrollo comunitario socioeconómico (HCRF, 2008: 8).

Desde la perspectiva del pueblo garífuna, el turismo es una oportunidad pero también es una posible amenaza, en especial por el impulso de grandes proyectos turísticos. ${ }^{1}$ Lo usual en países en vías de desarrollo es que, debido a su estructura (intensidad de know-how y capital), tanto los grandes proyectos turísticos como determinadas clases de turismo tienden a excluir a la población local de su control y manejo (Moreno, 2005: 236-240). En América Latina, los resorts (complejos hoteleros "todo incluido") y el turismo de cruceros tienen considerables consecuencias sobre la estructura de la oferta de productos y servicios extrahoteleros en los destinos (Pérez et al., 2003: 96-97).

Los intereses cognoscitivos de la presente investigación son:

- Caracterizar los diferentes tipos de turismo que se practican en el mnмcc.

- Estimar el grado de aceptación de los reality shows en las comunidades de influencia del mNMcc.

- Identificar los beneficios que resultan del turismo para la HCRF y las comunidades locales. 
Analizar si la política turística de la HCRF cumple con los objetivos de conservación ambiental y cultural estipulados para el mNMcc.

\section{Metodología}

El estudio se basa en datos cualitativos y cuantitativos obtenidos por el autor en Honduras entre febrero del 2013 y abril del 2014. Con base en un guion, se hicieron 15 entrevistas cualitativas con representantes de los patronatos, cooperativas de pescadores, pescadores independientes, turoperadores y funcionarios de la HCRF.

Para cuantificar la percepción y la aceptación del desarrollo turístico se llevó a cabo una encuesta de hogares en las comunidades garífunas del MNMcc. Las encuestas se realizaron de forma sistemática en uno de cada cinco hogares en las comunidades de Sambo Creek $(n=90)$ y Nueva Armenia $(n=60)$. Debido a la pequeña base poblacional y a su carácter transitorio, no era viable una encuesta sistemática en las comunidades isleñas. A diferencia de las comunidades insulares y Nueva Armenia, el porcentaje de mestizos de la población total de Sambo Creek es superior a $50 \%$. Por el enfoque específico del estudio y dada la importancia de los caladeros para las comunidades garífunas, también en Sambo Creek se encuestaron primordialmente garífunas. Para obtener una muestra representativa, las encuestas se efectuaron en zonas donde se concentra la actividad turística, en las cuales existen beneficios inmediatos; pero también en zonas marginales, con un mayor porcentaje de personas que no participan de manera directa en la actividad turística, o que de algún modo están excluidas de ella.

La encuesta recopiló datos personales (nombre, edad, sexo, nivel educativo, profesión) y estuvo dividida en dos partes. La primera incluye preguntas sobre las condiciones generales de vida en la comunidad: satisfacción de las necesidades básicas, servicios de salud y educación, actividades económicas, etc. La segunda parte abarca una serie de manifestaciones que el encuestado debe evaluar según el esquema de respuesta: "no sé”, "estoy de acuerdo", "no estoy de acuerdo”. Considerando que una porción significativa de la población tiene problemas de alfabetización, y para asegurar que las preguntas fueran comprendidas, se leyeron las preguntas a la persona encuestada. Con frecuencia, 
esto se convirtió en una entrevista cualitativa de la cual se obtuvieron conocimientos adicionales de trascendencia para el estudio.

Los datos empíricos se enriquecieron con otros estudios, en particular Brondo (2013), Brondo y Bown (2011) y Bown (2010). Bown analiza la dimensión del impacto ecológico y socioeconómico del comanejo en el mNMcc. Brondo aborda la temática del turismo y la filmación de reality shows en el MNMCc en relación con los derechos sobre la tierra, la represión y el acceso a recursos naturales. De gran relevancia para la discusión es el Plan de Manejo del Monumento Natural Marino Archipiélago Cayos Cochinos, donde la HCRF formula objetivos concretos de conservación e instrucciones operativas. En este sentido, completa las declaraciones de las entrevistas con representantes de la HCRF.

Tras esbozar el origen y la historia del pueblo garífuna se presentarán el área de estudio y el contexto turístico en el mnмcc. Con base en la información empírica de las encuestas y entrevistas se analizarán las dinámicas del desarrollo turístico y sus implicaciones para las comunidades garífunas del archipiélago. Por último, se resumen y se discuten los resultados obtenidos.

\section{El pueblo garífuna}

El pueblo garífuna nació en la isla de San Vicente en las Antillas Menores; surgió del mestizaje entre caribes arahuacos -que habían llegado desde la cuenca del Amazonas en América del Sur- y cimarrones africanos, que naufragaron 0 que se escaparon de las plantaciones en las islas de los alrededores (González, 2008: 60).

Por su continua resistencia guerrera el pueblo garífuna representó una amenaza permanente para el sistema colonial y esclavista europeo, lo cual motivó que, en 1797, después de haber sido derrotados en San Vicente, fueran deportados por los ingleses y abandonados en la isla de Roatán en la actual Honduras. Debido al inhóspito entorno de la isla, que les hizo más difícil la supervivencia, poco después de su llegada se trasladaron a tierra firme (González, 2008: 81), donde fundaron comunidades a lo largo de la costa caribeña de Centroamérica, en las orillas del mar y a lo largo de los ríos (Centeno García, 2001: 66). En la actualidad un número considerable ha migrado a Estados Unidos. 
"[R]esultado de esta historia viajera”, destaca Izard, es "una amalgama de elementos caribes, africanos, afroamericanos, europeos” (2010: 207). En 2001, la cultura garífuna fue declarada por la Unesco patrimonio intangible de la humanidad. Si bien hasta el día de hoy los garífunas siguen siendo sujetos de discriminación y racismo en la sociedad hondureña, muchos de ellos se han adaptado e integrado a la sociedad mestiza y extranjera, penetrando en las clases medias y altas, lo que significa en gran medida una pérdida de su cultura (England y Anderson, 1998: 8).

Para los garífunas el mar es mucho más que una fuente de alimentos. Es la base de su economía de subsistencia y un elemento esencial dentro de su cosmovisión. El mar es: "símbolo, expresión y testigo del desarraigamiento, de la enseñanza, del amor, del sufrimiento y de la liberación del pueblo garífuna” (Meléndez, 2010: 53).

Antes de que el archipiélago se declarara un área protegida, grupos de pescadores garífunas se habían asentado permanentemente en los Cayos Cochinos, haciendo uso de sus recursos marinos (Brondo y Bown, 2011: 94). La Smithsonian Institution, a la que primero se otorgó el manejo del área protegida (1993-1997), adoptó una política de expulsión hacia las comunidades, lo que coincidió con una militarización de la zona y una criminalización de la actividad pesquera garífuna. En efecto, la responsabilidad y la culpa por el deterioro de los recursos naturales marinos se atribuyeron a los garífunas que practican la pesca artesanal, utilizando cayucos de vela y técnicas tradicionales de cordel y el buceo al pulmón, pese a los evidentes daños provocados por la pesca industrial sobre los recursos pesqueros de la zona (Brondo, 2013: 138). A pesar de esta política conservacionista hostil, se logró la titulación definitiva de los cayos Chachahuate (Nueva Armenia) y Bolaños (Sambo Creek) y de la comunidad de East End en el Cayo Mayor (Río Esteban) en 2002 (Álvarez et al., 2013: 23).

\section{El contexto turístico}

En los últimos diez años el número de llegadas de turistas al mNMcc se ha incrementado notablemente (gráfica 1). 
Total de visitantes,

2008-2012

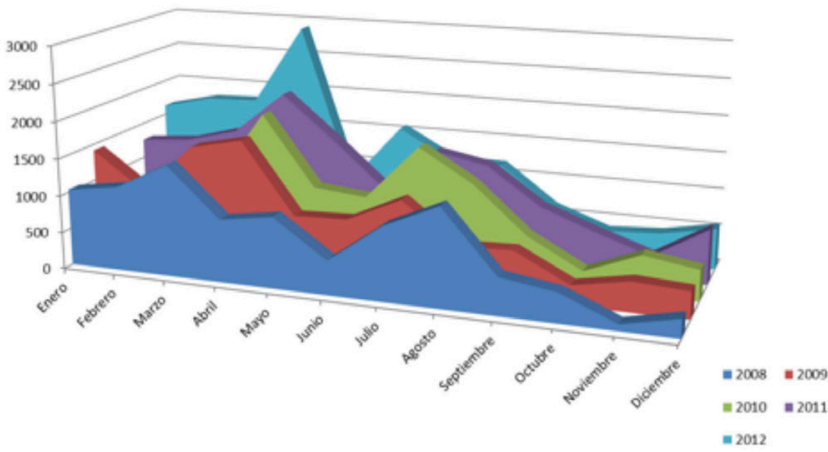

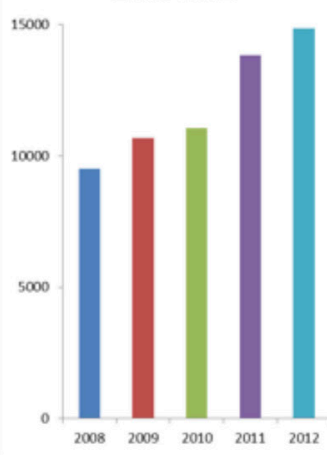

Fuente: Reunión para la conformación de la Asociación de Tour Operadores de Cayos Cochinos (elaboración propia).

Gráfica 1. Llegadas de turistas al mnmcc (2008-2012)

En el periodo de 2008 a 2012 el volumen de visitantes fue de 59887 personas y en él se vio un incremento de 56 \% (de 9505 en 2008 a 14833 en 2012). De manera señalada, en la Semana Santa del 2014, un total de 3000 personas llegaron al área protegida. ${ }^{2}$ Es de hacer notar que no todos quienes que visitan el área se registran ante la HCRF, y por ello esta cifra puede ser aún mayor. Se observa, además, una fuerte estacionalidad de la demanda, que se concentra en los meses de junio a agosto (coincidente con los periodos vacacionales en los principales países emisores de turistas) y en Semana Santa, considerada la temporada alta para el turismo nacional. En el pasado, los Cayos Cochinos fueron visitados sobre todo por extranjeros (Courrau y Andraka, 2004: 10), en la actualidad entre 80 y $90 \%$ de los visitantes al área son hondureños, gracias a una mejor promoción del destino en los medios nacionales (Locandro, 2010).

Es menester señalar que, pese a que existen iniciativas comunitarias en Chachahuate y East End, la HCRF fomenta de forma explícita un turismo de día y estimula hospedaje y alimentación en las comunidades costeras, en particular en Nueva Armenia, para asegurar una operación turística de bajo impacto y no incentivar la expansión de la infraestructura turística existente. ${ }^{3}$ La HCRF

${ }^{2}$ Entrevistas con representantes de la HCRF, La Ceiba, abril de 2014.

${ }^{3}$ Entrevistas con representantes de la HCRF, La Ceiba, abril de 2014; HCRF, 2008: 80. 
restringe a tres noches la estadía de los turistas en la zona. No obstante, no dispone de registros en cuanto a la ocupación de los hoteles. ${ }^{4}$

Recientemente, se ha registrado un sustancial crecimiento en llegadas de viajeros independientes, quienes se hospedan en las comunidades del archipiélago o en uno de los cayos privados y que por lo común permanecen más tiempo en el mNMcc, debido a una mayor promoción internacional del destino en las guías turísticas.

Con el fin de promover oportunidades para el desarrollo turístico en las comunidades locales, mediante el Decreto 114-2003, se implementó en 2004 el pago de un derecho de entrada (cuadro 1). Asimismo, gracias a este pago por servicio ambiental, la HCRF consideró esencial estructurar, controlar el volumen de personas y facilitar el acceso de turistas nacionales al mNMcc, con fines educativos y recreativos. Todo lo anterior expuesto con el propósito de promover la autosustentabilidad de la HCRF y de las comunidades (Denning, 2005: 139-141).

Tanto los turoperadores como los viajeros independientes tienen la obligación de reportarse en la estación científica para efectuar el pago de admisión

Cuadro 1. Derecho de admisión

\begin{tabular}{|c|c|c|c|}
\hline & $\begin{array}{l}\text { Un día } \\
\text { (USD*) }\end{array}$ & $\begin{array}{l}\text { Un mes } \\
\text { (USD) }\end{array}$ & $\begin{array}{l}\text { Un año } \\
\text { (USD) }\end{array}$ \\
\hline Visitantes extranjeros & 10 & 20 & 40 \\
\hline $\begin{array}{l}\text { Visitantes extranjeros } \\
\text { con turoperador }\end{array}$ & 5 & 10 & 20 \\
\hline Visitantes nacionales & 2 & 4 & 8 \\
\hline $\begin{array}{l}\text { Capitanes, guías, } \\
\text { habitantes }\end{array}$ & N.A. & N.A. & N.A. \\
\hline
\end{tabular}

Fuente: Elaboración propia con base en datos de anuncio público en la estación científica de Cayo Menor (2014).

\footnotetext{
${ }^{4}$ Entrevistas con representantes de la HCRF, La Ceiba, abril de 2014.
} 
antes de iniciar su recorrido. Suponiendo que en el periodo de 2008 a 2012 el total de turistas (59 887) que entraron al área hubiera sido de origen nacional (y que por ello pagaron dos dólares cada uno), podría estimarse que el ingreso de la HCRF fue de mínimo 119774 de dólares. Las comunidades turísticas del MNMCC, sin embargo, no participan en los ingresos generados por este concepto. ${ }^{5}$

Todos los turistas, al entrar, se registran en la estación científica, donde reciben un charla y se les muestra un video de carácter educativo enfocado en la biodiversidad, en la sensibilidad ecológica del archipiélago y en el concepto del turismo "sin dejar rastros", proporcionándoles códigos concretos de conducta para su estadía en el área protegida. Con todo, últimamente se observa que estas instrucciones son tomadas de forma irregular.

\section{Excursiones}

Más de la mitad de los turistas que visitan el archipiélago optan por un tour organizado de un día. Estas excursiones siguen un horario y un circuito establecidos, que incluyen la visita de los principales sitios de interés. Además de transporte, guía y asistencia personal, los paquetes pueden comprender servicios de comida y bebida y la facilitación de equipos para buceo, snorkel y pesca, entre otros.

En las comunidades del mNMcc hay un total de ocho turoperadores ${ }^{6}$ y un significativo número de individuos que ofrecen viajes a los Cayos Cochinos de forma independiente y oportuna. El servicio varía en calidad, volumen y naturaleza, normas de seguridad y estado de las embarcaciones según proveedor y punto de salida. De tal manera, puede concluirse que no hay un manejo estructurado ni organizado de la operación turística, lo que representa un gran desafío -ya identificado en el primer Plan de Manejo para el área (HCRF, 2004: 24)-; esto, pese a reconocer los esfuerzos de la HCRF por crear una asociación de turoperadores para estructurar y reglamentar la operación turística en el MNMCC (conforme a normas de seguridad y de calidad) y para reducir los daños causados por la actividad turística (Pineda y Aronne, 2012: 15-20) así como aumentar la competitividad de los pequeños operadores de servicios turísticos (HCRF, 2013). 
La comunidad de Sambo Creek es el punto de salida más importante. Otras excursiones al mnмcc salen de Nueva Armenia, La Ceiba y Roatán, siendo de menor jerarquía las comunidades de Río Esteban y Corozal. Debe señalarse que los itinerarios son poco diversificados. La mayoría incluye una parada para el almuerzo en Chachahuate, donde se tiene la oportunidad de comprar artesanías.

Tourist Options es un turoperador no garífuna con sede en la ciudad de La Ceiba y una sucursal en Sambo Creek, con servicios de hotel y restaurante. Dispone de una amplia gama de excursiones y traslados en Honduras y otros países de Centroamérica, y fue uno de los primeros en ofrecer paseos al MNMcc. En la actualidad ha llegado a controlar gran parte del mercado gracias a modernas embarcaciones y a una eficiente logística y promoción. La compañía también proporciona el servicio a los clientes de los hoteles Palma Real, Canadien y Helen en los alrededores de La Ceiba. ${ }^{7}$ Así, por ejemplo, cuenta con presencia en línea y, adicionalmente, es mencionado en eficaces guías como Honduras Tips y Lonely Planet. Por otro lado, Tourist Options se ostenta como un turoperador autorizado por la HCRF para moverse dentro del área protegida. Desde La Ceiba, un tour a los Cayos Cochinos con Tourist Options cuesta 40 dólares por persona (mínimo seis personas), e incluye el transporte terrestre al Centro Turístico en Sambo Creek, transporte marítimo, guía, entradas y equipo de snorkel (Tourist Options, 2014). Las embarcaciones tienen una capacidad de entre 16 y 21 personas, y la tripulación está compuesta por un capitán, un ayudante y el guía. En el precio no están incluidos el almuerzo ni otros gastos personales.

En Roatán, varios hoteles y agencias ofrecen viajes a los Cayos Cochinos. El Infinity Bay Resort, por ejemplo, vende el paquete turístico por 180 dólares por persona, que cubren bar abierto, almuerzo, bocadillos y equipo de snorkel; dura ocho horas y el destino es la comunidad garífuna de Chachahuate. El Infinity Bay Resort promueve la excursión a los Cayos Cochinos como un viaje a las últimas islas desiertas e intocadas en el Caribe. En el recorrido se menciona a los garífunas, los habitantes de estas islas, que "viven del agua de lluvia y pescado fresco en pequeñas cabañas con techo de paja” (Infinity Bay Spa, 2014: 4). En esencia, los anuncios acentúan la belleza natural (playas, arrecifes, etc.) de los Cayos Cochinos y la soledad donde los pescadores garífunas "invitan a los turistas a convivir y conocer su estilo de vida y degustar sus comidas" (Hyde Tours Roatan, traducción libre del inglés por el autor).

${ }^{7}$ Entrevista con Mauricio Elvir, gerente general del Centro Turístico, Sambo Creek, 2 de junio de 2013. 


\section{Turismo SAVE}

El turismo científico, académico, voluntario y educativo (save, por su siglas en inglés) ha sido identificado como un valioso nicho de mercado para la costa norte y las Islas de la Bahía de Honduras. Los Cayos Cochinos han sido seleccionados por el entorno natural "no desarrollado y prístino" (GWU/IHT, 2003: 26), la presencia y el potencial turístico de las comunidades garífunas y las oportunidades para exploraciones científicas marinas y etnográficas. La HCRF dispone de instalaciones en la estación científica del Cayo Menor para realizar investigaciones (GWU/ıнт, 2003: 28). Es pertinente señalar que el turismo voluntario en el MNMCc está vinculado a la presencia de ong ambientalistas internacionales, que llevan a cabo proyectos y programas de conservación en concertación con la HCRF. Los estudiantes voluntarios trabajan bajo la supervisión de investigadores experimentados.

El turismo voluntario obtuvo mayor peso en el mNMcc a partir del 2003, cuando se institucionalizó la colaboración de la HCRF con la Operación Wallacea (Opwall) (organización científica británica con fines de lucro), por un periodo de diez años (Brondo, 2013: 130). De las organizaciones que han trabajado en el MNMcc la Opwall es la más importante y la que mayor impacto ha tenido sobre el área. Esto se refleja en su periodo de permanencia y en el número de estudios e investigaciones realizadas. Opwall ejecuta sus programas de conservación e investigación a través de una ong anfitriona local, minimizando los efectos negativos sobre el medio ambiente, la cultura y las costumbres de las poblaciones locales, y maximizando los beneficios para las comunidades mediante el desarrollo de fuentes alternativas de ingresos (Brondo y Woods, 2007: 6; Brondo, 2013: 156). La organización sostiene sus proyectos y actividades de conservación a través de cuotas que los investigadores y estudiantes voluntarios pagan para participar en ellos (Brondo/Bown, 2011: 95). El equipo de investigadores permanece largas temporadas en el MNMcc y lleva a cabo actividades referentes a la conservación, el monitoreo y la capacitación de la población local (Courrau y Andraka, 2004: 7). En los meses de junio a agosto de 2006 llegaron aproximadamente 1200 estudiantes voluntarios de la Opwall al mNMcc. Entre 2004 y 2005 el número de estudiantes británicos en la zona era aún más alto. A partir del 2006, una vez que la HCRF y Opwall renegociaron el contrato, se redujo de manera drástica este volumen (Brondo y Woods, 2007: 15). La HCRF, la cual recibe 
una parte significativa de dicha cuota, ha sido beneficiada en términos económicos por la asociación, sobre todo por la facilitación de servicios específicos como alojamiento, alimentación y la logística del transporte que contribuyen al éxito de la operación, hasta tal punto que se consideró la principal fuente de ingresos de la organización (Brondo y Woods, 2007: 3).

Al ser un elemento integral de la política y del compromiso social de la organización, la Opwall pretendió tener un impacto económico positivo en las comunidades locales, coordinando y contratando mano de obra y servicios de transporte, alojamiento y alimentación directamente en las poblaciones locales (Brondo y Woods, 2007: 6; Denning, 2005: 13). Sin embargo, en los primeros dos años de su operación en el mNMcc (2004-2005), los grupos de investigadores y estudiantes de Opwall se instalaron exclusivamente en la estación científica de la HCRF en el Cayo Menor, por lo que el nivel de interacción con las comunidades y los beneficios tangibles para ellas fueron mínimos. La HCRF les prestaba la logística diaria de alojamiento, alimentación y transporte, dejando de lado por completo a las comunidades garífunas. Para alojar a estudiantes e investigadores, con el apoyo financiero de la Organización Mundial para la Naturaleza (wwF por sus siglas en inglés), se construyeron cabañas en la estación científica.

Es de reconocerse que se otorgó una alta prioridad a los estudios marinos, a diferencia de investigaciones de carácter sociocultural, por lo menos hasta 2007. Entre 2007 y 2009 se integraron varios programas enfocados en las condiciones de vida de las comunidades del archipiélago (Brondo, 2013: 158). Tras la creación de una unidad móvil de investigación social en 2006, la base de los investigadores y estudiantes del área social se trasladó del Cayo Menor hacia las comunidades garífunas costeras, primero a Nueva Armenia (2006-2007) y después a Río Esteban (2008-2011). Para apoyar el desarrollo turístico comunitario, la organización coordinaba el alojamiento y la alimentación en casas particulares de familias. Las comunidades reciben un máximo de 200 estudiantes por semana. Con todo, los beneficios no fueron distribuidos de modo equitativo en el plano comunitario, debido al número limitado de hogares aptos para alojar a los estudiantes (Brondo y Bown, 2011: 98).

En el marco de la estrategia para el desarrollo socioeconómico comunitario de diferentes organizaciones se ha puesto un mayor énfasis en la promoción del turismo en relación con la construcción y el mejoramiento de la infraestructura 
turística existente en el archipiélago. Asimismo, el personal y los estudiantes voluntarios de Opwall implementaron una serie de actividades regulares, tales como excursiones a la comunidad de Chachahuate, presentaciones de danza garífuna y otros con el fin de desarrollar el turismo y generar fuentes de empleo e ingresos directos en las comunidades (Young et al., 2006: 9). En gran parte estos eventos fueron puestos en práctica para entretener a los mismos estudiantes de la organización. Las ganancias fueron donadas sobre todo a la escuela de East End. A pesar de esto, en 2006 se suspendieron las excursiones a Chachahuate y otras actividades sociales como reacción a un caso de robo ocurrido durante un evento en el cual estudiantes de la Opwall y pobladores garífunas estuvieron presentes (Young et al., 2006: 9-10; Brondo, 2013: 160).

La cooperación a largo plazo con la Opwall resultó en un flujo constante de estudiantes e investigadores al MNMcc y representó la principal fuente de ingresos para la HCRF (Brondo y Woods, 2008: 3). La retirada de esta organización a finales del 2013, en consecuencia, tiene amplias repercusiones para el funcionamiento económico de la HCRF, pues, de haberse renovado el contrato esta hubiera tenido garantizados sus ingresos; pero la Opwall no estaba dispuesta a firmar otro acuerdo y trasladó sus actividades a la bahía de Tela, con el fin de prestar asistencia para el establecimiento de una nueva área protegida marina.

\section{La filmación de reality shows}

En 2006 se introduce un nuevo fenotipo de la actividad turística en el MNMcc: la filmación de reality shows. Entre 2006 y 2014, televisiones de Italia, España, Ucrania y Colombia han grabado 12 reality shows (cuadro 2). Estos programas llegan a tener cuotas de pantalla de entre 4 y 11 millones de televidentes en los países emisores, lo que corresponde a hasta 46 \% del mercado (Morales, 2014).

La filmación de reality shows en el MNMcc se considera parte de la actividad turística en especial por dos razones:

Durante la estadía, tanto los concursantes como el equipo de grabación consumen una serie de productos y servicios turísticos que contribuyen al éxito de la operación. Entre los más señalados se pueden mencionar alojamiento, alimentación y transporte.

La emisión de los reality shows en los respectivos países eventualmente tiene un impacto positivo sobre la demanda de viajes a los países donde se filman 
CUAdRo 2. Filmación de reality shows en el mnmcc según país y año

\begin{tabular}{|c|c|c|c|c|c|c|c|c|c|c|}
\hline Programa & País & 2006 & 2007 & 2008 & 2009 & 2010 & 2011 & 2012 & 2013 & 2014 \\
\hline L'Isola dei Famosi & Italia & $\begin{array}{l}13 \text { sep.- } \\
01 \text { nov. }\end{array}$ & $\begin{array}{l}19 \text { sep.- } \\
28 \text { nov. }\end{array}$ & $\begin{array}{l}15 \text { sep.- } \\
24 \text { nov }\end{array}$ & $\mathrm{X}$ & $\mathrm{X}$ & $\begin{array}{l}14 \text { feb.- } \\
26 \text { abril }\end{array}$ & $\begin{array}{l}25 \text { ene.- } \\
05 \text { abril }\end{array}$ & $\mathrm{X}$ & \\
\hline $\begin{array}{l}\text { Supervivientes: } \\
\text { Perdidos en } \\
\text { Honduras }\end{array}$ & España & $\mathrm{X}$ & $\begin{array}{l}19 \text { abril- } \\
28 \text { jun. }\end{array}$ & $\begin{array}{l}17 \text { ene.- } \\
27 \text { mar. }\end{array}$ & $\begin{array}{l}19 \text { mar.- } \\
11 \text { junio }\end{array}$ & $\mathrm{X}$ & $\begin{array}{l}05 \text { mayo- } \\
28 \text { julio }\end{array}$ & $\mathrm{X}$ & $\mathrm{X}$ & $\begin{array}{l}17 \text { mar.- } \\
27 \text { mayo }\end{array}$ \\
\hline $\begin{array}{l}\text { La isla de los } \\
\text { famosos-Una aventu- } \\
\text { ra maya }\end{array}$ & Colombia & $\mathrm{X}$ & $\begin{array}{l}13 \text { mar. } \\
37 \text { episo- } \\
\text { dios }\end{array}$ & $\mathrm{X}$ & $\mathrm{X}$ & $\mathrm{X}$ & $\mathrm{X}$ & $\mathrm{X}$ & $\mathrm{X}$ & \\
\hline Останни́ Герои́ & Ucrania & $\mathrm{X}$ & $\mathrm{X}$ & $\mathrm{X}$ & $\mathrm{X}$ & $\mathrm{X}$ & $\mathrm{X}$ & sep-oct. & $\mathrm{X}$ & \\
\hline
\end{tabular}

Fuente: Elaboración propia con datos de Magnolia tv, Telecinco

< http://www.telecinco.es/supervivientes/ > , rai 2, rcn y ictv.

estos programas. Lo cual, a su vez, puede facilitar la creación de una oferta muy especializada que se concentra en visitar los escenarios cinematográficos reales. Nueva Zelanda, por ejemplo, registró un incremento significativo en llegadas de turistas por las imágenes paisajísticas y el éxito comercial de la trilogía de El Señor de los Anillos.

De conformidad con este punto de vista, la HCRF ha autorizado las grabaciones en el área protegida de Cayos Cochinos argumentando:

El potencial de crear oportunidades de empleo en las comunidades locales. El efecto de publicidad que se espera de las filmaciones para el destino turístico Cayos Cochinos.

En el Plan de Manejo del área se destaca que la filmación de reality shows se permite solo "bajo una reglamentación especial [...], conservando el espíritu de protección primario de la zona” (HCRF, 2008: 138).

Es crucial notar que la HCRF le brinda la logística diaria al equipo del reality show y actúa como intermediador, contratando y coordinando todos aquellos servicios que no puede proporcionar. ${ }^{8}$

${ }^{8}$ Entrevistas con representantes de la HCRF, La Ceiba, abril de 2014, y representantes de las comunidades, marzo y abril de 2014 . 


\section{Oportunidades laborales}

Potencialmente, existen nuevas oportunidades de empleo para los sectores de alojamiento, alimentación y de transporte terrestre y marítimo tanto de los candidatos como del equipo de la televisión. Se puede observar, empero, que los puestos de trabajo creados se asocian en su mayoría con la logística del transporte, es decir con el traslado de los concursantes y del equipo dentro del MNMCc, y es la HCRF la que, con sus propias embarcaciones, efectúa el transporte diario del equipo entre el Hotel Palma Real en tierra firme y el MNMcc. ${ }^{9}$

Pese a que hay varios lancheros, en particular en Nueva Armenia, que trabajan en el reality show y que han sido contratados por la HCRF, muchas veces la población local se ve excluida de este tipo de trabajos por falta de capital de inversión propio. En las comunidades son unas cuantas personas quienes disponen de embarcaciones aptas para ser contratadas para las filmaciones.

En lo que se refiere al alojamiento, dos factores son decisivos: la calidad y la capacidad de los hoteles. En todas las comunidades garífunas del MNMCC existen cabañas u hoteles sencillos y económicos. Las comunidades carecen de infraestructura turística adecuada que atienda este tipo de turismo, pues todos estos alojamientos no disponen ni de las capacidades para albergar al equipo del reality show (hasta 200 personas) ni cumplen con los estándares que se esperan para este clase de operaciones. El equipo se hospeda en el Hotel Palma Real, un complejo hotelero que antes pertenecía a la cadena española Barceló, ubicado a $20 \mathrm{~km}$ de la ciudad de La Ceiba. Asimismo, la HCRF pone a disposición en el Cayo Menor las cabañas que antes eran de uso exclusivo de los investigadores y estudiantes extranjeros de la Opwall. Los servicios extrahoteleros de alimentación son proporcionados por la HCRF en el Cayo Menor.

Los garífunas han sido empleados para la instalación submarina de cables y otros trabajos de instalación y construcción, al igual que en la cocina de la HCRF en el Cayo Menor. La productora de televisión ofrece puestos técnicos no cualificados (asistente de cámara o de sonido, por citar algunos). En otro momento, pobladores garífunas fueron contratados para un ensayo piloto antes del inicio de las grabaciones; fueron filmados sin acuerdo previo y recibieron a cambio

\footnotetext{
${ }^{9}$ Entrevistas con lancheros, noviembre de 2013, y representantes de la HCRF, La Ceiba, abril de 2014.
} 
una remuneración de diez dólares. ${ }^{10}$ Este tipo de personal ha sido contratado directamente por las empresas, sobre todo en la comunidad de Sambo Creek. ${ }^{11}$ A la luz de las declaraciones de la HCRF acerca de que las filmaciones iban a beneficiar a las comunidades garífunas, los líderes de las comunidades critican la falta de consideración de garífunas preparados para trabajos de mayor prestigio y remuneración. ${ }^{12}$

\section{Restricciones}

A pesar de que puede verse una clara diversificación de trabajos en las comunidades y la integración de actividades económicas no tradicionales, como por ejemplo el turismo, aún hay muchas personas que directa o indirectamente dependen de la pesca. Desde que se creó el mnмcc se han puesto en marcha una serie de normas y restricciones que afectan en gran medida las rutinas de trabajo de los pescadores artesanales locales. ${ }^{13}$ Esta política conservacionista ha coincidido con una criminalización de la actividad pesquera garífuna junto con una represión armada ejecutada por las fuerzas navales que patrullan la zona. Una vez dada una infracción, se adoptan medidas disciplinarias que, además de elevadas multas, en caso de reincidencia prevén la confiscación de las embarcaciones, la base de la pesca. ${ }^{14}$

El contrato que la HCRF suscribe con las productoras de televisión establece que, para no alterar la imagen del ficticio naufragio en el mar Caribe, los pescadores garífunas no pueden acercarse al respectivo cayo donde se esté grabando. Durante las filmaciones, las restricciones se amplían por igual a otras áreas del MNMCC.

En 2014 el reality show español Supervivientes: Perdidos en Honduras se filmó en los cayos Timón y Paloma. Tradicionalmente, las aguas en los alrededores de este último han sido un sitio fundamental para la captura de "fra", una carnada que sirve para la pesca de peces más grandes. Con el argumento de la

${ }^{10}$ Entrevista con el Patronato de Sambo Creek, marzo de 2014.

${ }^{11}$ Entrevista con representantes de la HCRF, La Ceiba, abril de 2014.

${ }_{12}$ Entrevistas con los patronatos de Nueva Armenia y Sambo Creek, febrero y marzo de 2014.

${ }^{13}$ Entrevistas con representantes de la Asociación de Pescadores Santa Ana, marzo de 2014, y pescadores independientes.

${ }^{14}$ Entrevistas con pescadores en el mNMcc, diciembre de 2013; Brondo, 2013: 14. 
conservación de la biodiversidad, desde la creación del área protegida, no se les ha permitido a los pescadores garífunas el acceso a este cayo (Brondo, 2013: 150). La HCRF, la propietaria del cayo Paloma, lo arrienda a las compañías televisoras por el tiempo que duren las filmaciones, pero es un sitio de reproducción de aves y de tortugas (HCRF, 2008: 59), siendo esta la principal razón por la cual el acceso ha sido restringido. El Plan de Manejo estipula que:

[e]n las épocas de reproducción [...] de aves marinas, entre el 1 de abril al 30 de mayo y el 1 de julio al 30 de septiembre, en Cayo Timón, Paloma [...] se evitará perturbarlas con la actividad de extracción de carnada [HCRF, 2008: 134].

En contradicción con este señalamiento, se toleran las grabaciones en los cayos Timón y Paloma precisamente en estas épocas de reproducción, garantizando a los concursantes plenos derechos de pesca en la zona (Brondo y Bown, 2011: 91). Ante los ojos de la HCRF los concursantes capturan langostas, una especie para la cual se aplican restricciones de pesca que abarcan, además de límites de tallas, una veda estacional entre marzo y agosto (HCRF, 2008: 134). Por la importancia alimentaria de la langosta y por su valor comercial, la puesta en práctica de vedas es una medida que las comunidades no aceptan (HCRF, 2008: 39). La impunidad de la que los concursantes gozan dificulta a su vez la imposición y el razonamiento de las normas en las comunidades garífunas pesqueras. Además, los pescadores son obligados a recorrer cada vez mayores distancias para capturar lo suficiente y, dado que no utilizan embarcaciones motorizadas sino cayucos de remo o vela, corren el riesgo de ser afectados por fenómenos meteorológicos.

En la actualidad, para compensar lo que dejan de ganar al no pescar, las productoras televisoras pagan mil dólares a cada una de las cinco comunidades afectadas. El pago, no obstante, no se otorga directamente a las comunidades sino que es canalizado mediante la HCRF que descuenta 250 dólares por gastos de reuniones y capacitaciones realizadas con las comunidades, previas a las filmaciones. El reembolso del importe restante se realiza solo vinculado con proyectos comunitarios. En lo que concierne al uso de este dinero, los líderes de las comunidades señalan las posibilidades limitadas de realizar proyectos de mayor dimensión para el bienestar comunitario. El dinero ha sido utilizado para pequeños proyectos, entre ellos la reparación de un puente en Nueva Armenia y 
para las escuelas comunitarias. ${ }^{15}$ Como un elemento tangible de su compromiso social, al finalizar el rodaje las productoras de televisión suelen regalar medicamentos y equipo excedente a sus empleados locales. ${ }^{16}$ Brondo (2013: 149) estima las ganancias que resultan de las filmaciones para la HCRF en 560000 dólares.

Con todo y que las comunidades reclaman un mayor protagonismo y participación (económica) en las decisiones en materia de las filmaciones, las negociaciones entre la HCRF y las productoras de televisión no se han hecho más transparentes y no se les permite la participación de representantes del pueblo garífuna.

\section{Distribución de ganancias}

La distribución de los beneficios económicos generados por la filmación de reality shows en el mNMcc ha sido desequilibrada. En Nueva Armenia, $88 \%$ de los encuestados respondió que la comunidad no se ha beneficiado de las filmaciones. En Chachahuate $62.5 \%$ y en East End $60 \%$ opinan que los beneficios han sido escasos o ausentes. La imagen que presenta Sambo Creek es distinta: $69 \%$ de los encuestados contestó que la comunidad ha sido beneficiada por las filmaciones.

A pesar de lo anterior, $83 \%$ de los encuestados no cree que debería prohibirse la filmación de reality shows en el MNMCc. El rechazo se dirige a la HCRF que, según las comunidades, se apodera de la mayor parte de los ingresos, por lo cual se sienten discriminadas y explotadas, de ahí que, $80 \%$ de todos los encuestados indiquen que la HCRF favorece a empresas y personas de altos ingresos y discrimina a las comunidades.

\section{Futuras alianzas con empresa privada}

La asociación de la HCRF con la Fundación Azteca (el brazo social del Grupo Salinas, un conjunto de empresarios mexicanos) tiene el objetivo de, en el futuro,

\footnotetext{
${ }^{15}$ Entrevistas con los patronatos de Nueva Armenia y Sambo Creek, 4 de febrero de 2014 y 28 de marzo

${ }^{16}$ Ídem.
} de 2014. 
impulsar más el desarrollo turístico en el archipiélago. El involucramiento del Grupo Salinas en el mnмcc es interesante por dos razones. Primera, Televisión Azteca, perteneciente a este consorcio, es la productora de La isla, el formato mexicano del reality show de "supervivencia”, que se filmó en Nicaragua, entre otros países. Segundo, por el desarrollo con fines turísticos que hoy en día se está impulsando en Cayo Redondo, propiedad de empresarios hondureños, por parte de la empresa mexicana GVA (GVA, s. f.).

\section{El turismo de cruceros}

Pese a que el Plan de Manejo prohíbe el tránsito de cruceros (HCRF, 2008: 135), estos han llegado al MNMcc en varias ocasiones. Son buques de alto calado que, por lo tanto, pueden causar daños al arrecife. El barco se estaciona entre los cayos Mayor y Menor. Tourist Options organiza el traslado de los turistas a Chachahuate y otros sitios de interés.

Los grandes buques tienen una capacidad de hasta 6000 pasajeros y son definidas como ciudades flotantes con una oferta de servicios integrales. Así, el turismo de cruceros representa:

una combinación entre transporte, alojamiento y entretenimiento, uniendo en un mismo espacio flotante las infraestructuras necesarias para el desplazamiento, la estancia, el esparcimiento y los demás servicios destinados a los consumidores [Fernández Miranda, 2012: 56].

Esta integración vertical progresiva de servicios y productos turísticos limita los beneficios tangibles para las comunidades a la venta de artesanía y a presentaciones folklóricas. La HCRF, en cambio, recibe los pagos de derechos de admisión según el número de pasajeros y tripulantes.

Además, existe el proyecto de ampliar la oferta de servicios de transbordos marítimos regulares por conexiones entre las Islas de la Bahía (Roatán, Utila, Guanaja), La Ceiba y los Cayos Cochinos.17 En la actualidad solo se ofrece un transbordo directo entre las islas de Roatán y Utila, los destinos más turísticos del país, y la ciudad de La Ceiba.

17 Secretaría de Relaciones Exteriores, 2011: 4; entrevista con representante de Galaxy Wave Ferry, Roatán, 19 de diciembre de 2014. 


\section{Conclusión}

En vista de lo expuesto, la empresa privada y las organizaciones no gubernamentales -con y sin fines de lucro- frecuentemente compensan el vacío dejado por la ausencia del Estado y los escasos fondos públicos en cuanto al financiamiento y el manejo de áreas protegidas, de manera señalada en países en vías de desarrollo.

Dada la dependencia de fondos externos, la HCRF se ve obligada a comercializar el acceso a los recursos naturales del mnмcc para llevar a cabo sus programas y proyectos de conservación y, en consecuencia, ha establecido asociaciones con empresas privadas vinculadas a la industria de turismo, lo que a su vez ha resultado en una creciente interconectividad de los objetivos de conservación de los recursos naturales y el desarrollo de ellos a través del turismo, y esto con fines de lucro. En tal virtud, el desarrollo turístico en el mNMcc ha pasado de ser un medio para promover el desarrollo socioeconómico comunitario y reducir la dependencia de la pesca de las comunidades a una de las principales fuentes de ingresos para la HCRF. Los beneficios palpables para las comunidades garífunas, en cambio, son inferiores en comparación con el impacto negativo causado por el actual desarrollo turístico. A diferencia de la reivindicación de un turismo "sin dejar rastros", la HCRF tolera y promueve activamente el desarrollo turístico masivo, intensivo en capital y know-how, que tiende a excluir a las comunidades garífunas del control, restringiendo su aprovechamiento a la venta de comida y artesanías.

Esto es válido en especial en lo concerniente al turismo de cruceros y la filmación de reality shows. La promoción del turismo de crucero en el área y la concesión de permisos de filmación a las compañías televisoras en los cayos Paloma y Timón, durante los ciclos de reproducción de tortugas y aves marinas, entran en plena contradicción con los objetivos de conservación estipulados para el área. La HCRF aplica una política ambiental conservacionista de doble rasero, ya que la imposición legal de normas establecidas es selectiva y depende en gran medida de los intereses de quiénes estén involucrados. De acuerdo con las encuestas realizadas, $80 \%$ de los encuestados opina que la HCRF privilegia a personas con capital y discrimina a las comunidades. Desde el punto de vista de las productoras de televisión, las locaciones son intercambiables, lo que fortalece su posición al momento de las negociaciones. Se buscan países en 
vías de desarrollo no solo por sus paisajes sino también por los bajos costos de producción y la existencia de legislaciones débiles. Está claro que no es una coincidencia que la filmación de los reality shows se realice principalmente en sitios como Honduras, Nicaragua y República Dominicana, lo cual representa una continuación del debate sobre los mecanismos de la globalización.

El turismo científico-voluntario, en cambio, ha sido visto como una actividad deseable en las áreas protegidas y acorde con los fines de conservación. En el caso del MNMcc, sin embargo, se observó una masificación por lo menos en los primeros años de la asociación con Opwall, poniendo en duda la política y los objetivos de conservación propagados por ambas organizaciones. Como consecuencia de un robo ocurrido durante un evento de estudiantes de la Opwall, se les prohibió a los garífunas el acceso al Cayo Menor cuando los estudiantes estuvieran presentes, aunque no se demostró que algún garífuna haya sido culpable del hurto.

Es evidente que, además del tipo de turismo fomentado, el volumen de turistas es un factor que limita y repercute en la sostenibilidad del desarrollo turístico. Al igual que las otras clases de turismo, las excursiones muestran una fuerte estacionalidad que coincide con los periodos vacacionales de los principales países emisores de Europa y Norteamérica y de Honduras. La inauguración de un transporte marítimo regular entre Roatán, Utila y los Cayos Cochinos, sin duda, intensificará la presión sobre los recursos naturales y culturales del archipiélago. Chachahuate, la comunidad más turística del MNMcc, no dispone de la solvencia para absorber la dimensión del crecimiento turístico no controlado debido a sus características geofísicas y a las limitadas capacidades para resolver conflictos. El cayo carece de fuentes de agua potable, de energía limpia, de un sistema de saneamiento de aguas negras y de recolección de basura. En efecto, las condiciones de higiene son precarias y los conflictos sociales han aumentado por el turismo.

No obstante que la mayoría de las excursiones y cruceros que entran al MNMCc incluyen una parada en Chachahuate, la comunidad no participa en los ingresos generados por el pago obligatorio del derecho de admisión. La HCRF encauza estos ingresos, dejando de lado por completo a las comunidades garífunas. Al mismo tiempo, la HCRF le presta asistencia insuficiente a la comunidad en cuanto a la recolección y transportación de desechos inorgánicos a tierra firme y a la mediación de conflictos. 
En conclusión, se identificó que la distribución de los ingresos por la actividad turística ha sido desequilibrada y no transparente, beneficiando de manera primordial a la HCRF. En lo que concierne a los reality shows, la HCRF proporciona la logística diaria al equipo de filmación y dirige los pagos a las comunidades, cobrando comisiones. A la luz del gran éxito de estos programas, los pagos otorgados son muy bajos e insuficientes para financiar importantes obras públicas en las comunidades. Además, el reembolso no es directo sino vinculado con la realización de un proyecto comunitario concreto. Esto a su vez refuerza la imagen paternalista de la HCRF, reduciendo la autonomía económico-social de las comunidades, y cuestiona la capacidad de ellas de manejar estos fondos de un modo responsable.

Aunque las filmaciones crean nuevas oportunidades de empleo, este efecto se relativiza por trabajos tradicionales, en particular la pesca artesanal, que se ponen en riesgo por los reality shows de "supervivencia". Si bien es cierto que la pesca está perdiendo importancia como medio de vida y base histórica del sustento, y está siendo sustituida por actividades económicas como el turismo, aún hay muchas personas que directa o indirectamente dependen de ella y que, por lo tanto, se ven afectadas por la ampliación de restricciones durante las filmaciones. Por la imposición selectiva de las normas y el otorgamiento de amplias concesiones y una impunidad jurídica a los concursantes de los reality shows, queda en evidencia que se está aplicando una política de doble rasero que favorece a las personas que disponen de capital. Esto, por su parte, dificulta la implantación y el razonamiento de las normas en las poblaciones locales.

A futuro es fundamental que la HCRF revise sus estrategias y políticas de turismo para el área protegida, a fin de asegurar que las actividades tengan un beneficio tangible para las frágiles comunidades garífunas mediante una distribución más equitativa de los recursos financieros.

\section{Fuentes citadas}

Aderhold, P. (2000). Tourismus in Entwicklungsländer- Eine Untersuchung über Dimensionen, Strukturen, Wirkungen und Qualifizierungsansätze im Entwicklungsländer-Tourismus - unter besonderer Berücksichtigung des deutschen Urlaubsreisemarktes. Ammerland: Studienkreis für Tourismus und Entwicklung. 
Álvarez, C. et al. (2013). "Tierras y territorios de las comunidades garífunas y afrodescendientes de Honduras”. Revista Medio Ambiente, 1, 5-65.

Bown, N. (2010). "Contested Models of Marine Protected Area (MPA) Governance: A Case Study of the Cayos Cochinos, Honduras". Tesis de Doctorado. Newcastle: Universidad de Newcastle.

Brondo, K.V. (2013). Land Grab. Green Neoliberalism, Gender, and Garifuna Resistance in Honduras. Tucson: The University of Arizona Press.

Brondo, K.V. y N. Bown (2011). "Neoliberal Conservation, Garifuna Territorial Rights and Resource Management in the Cayos Cochinos Marine Protected Area”. Conservation and Society, 9 (2), 91-105.

Brondo, K.V. y L. Woods (2007). "Garifuna Land Rights and Ecotourism as Economic Development in Honduras' Cayos Cochinos Marine Protected Area”. Ecological and Environmental Anthropology, 3 (1), 1-18.

Centeno García, S. (2001). Historia del pueblo negro caribe y su llegada a las Hibueras el 12 de abril de 1797. Tegucigalpa: Universidad Nacional Autónoma de Honduras/Editorial Universitaria.

Courrau, J. y S. Andraka (2004). Manejo del turismo en el Monumento Natural Marino Archipiélago Cayos Cochinos, Honduras. Límites de cambio aceptable en la protección de los recursos naturales y las experiencias de los visitantes en zonas de uso turístico. San José: World Wildlife Fund Centroamérica (wwF Centroamérica).

Denning, P. (2005). “Interim Marine Field Report. Honduras Marine Site June-August 2005” [en línea]. Fundación Cayos Cochinos/Corporación Hondureña de Desarrollo Forestal. Disponible en: http://opwall.com/ wp-content/uploads/Interim_Marine_Field_Report_Honduras_2005. pdf [2014, 12 de diciembre].

England, S. y M. Anderson (1998). "Authentic African Culture in Honduras? Afro-Central Americans Challenge Honduran Indo-Hispanic Mestizaje”. Ponencia presentada en el XXI Latin American Studies Association International Congress, Chicago, 24-27 de septiembre.

Fernández Miranda, R. (2012). "Lo que hunden mientras flotan”. Alba Sud [en línea], 15 de noviembre. Disponible en: http://www.albasud.org/blog/ es/355/lo-que-hunden-mientras-flotan [2014, 8 de noviembre].

Friedl, H. (2002). Tourismusethik- Theorie und Praxis des umwelt- und sozialverträglichen Fernreisens. Múnich: Profil Verlag. 
gVA (s. f.). "Cayo Redondo. Cayos Cochinos Honduras" [en línea]. Disponible en: http://www.gva.com.mx/portafolio-detalle. php?tipo $=$ tipologia\&prjId $=31$

GwU/IHT (2003). "Accessing Opportunities in Scientific, Academic, Volunteer and Educational (save) Tourism in the North Coast and Bay Islands, Honduras" [en línea]. George Washington University-мBA Consulting Practicum/Instituto Hondureño de Turismo, Washington. Disponible en: http://rmportal.net/library/content/nric/1703.pdf [2014, 12 de diciembre].

González, N.L. (2008). Peregrinos del Caribe. Etnogénesis y etnohistoria de los garífunas. Tegucigalpa: Guaymuras.

HCRF (2004). Plan de manejo del Monumento Natural Marino Archipiélago Cayos Cochinos, Honduras 2004-2009. La Ceiba: wwF Centroamérica/Fundación Hondureña para la Protección y Conservación de los Cayos Cochinos.

------ (2008). Plan de manejo del Monumento Natural Marino Archipiélago Cayos Cochinos, Honduras 2008-2012. La Ceiba: Serviprensa.

------ (2013). "Realidades de la operación turística actual en Cayos Cochinos". Reunión para la conformación de la Asociación de Tours Operadores de Cayos Cochinos, Sambo Creek, 3 de diciembre.

Hyde Tours Roatan (s. f.). "Cayos Cochinos - Pigeon Key”, en Hyde Tours Roatan [en línea]. Disponible en: http://hydetoursroatanhonduras.com/ portfolio-items/cayos-cochinos-or-pigeon-cays/ [2014, 6 de noviembre].

Infinity Bay Spa \& Beach Resort (2014). Infinity Bay Magazine. S. 1.: s. e. Izard, G. (2010). "Garífuna y seminole negros: mestizajes afroindígenas en Centro y Norteamérica”, en E. Cunin (dir.). Mestizaje, diferencia y nación. Lo "negro" en América Central y el Caribe. México: Instituto Nacional de Antropología e Historia, 197-222.

Locandro, Y. (2010). “Los Cayos Cochinos, un paraíso catracho”. La Prensa [en línea], 24 de abril. Disponible en: http://www.laprensa.hn/honduras/501247-97/cayos-cochinos-un-paraiso-catracho

Meethan, K. (2002). Tourism in Global Society- Place, Culture, Consumption. Basingstoke: Palgrave Macmillan. 
Meléndez, A.C. (2010). Adeija Sisira Gererum Aguburigu Garinagu- "El enojo de las sonajas; palabras del ancestro”. Tegucigalpa: Guardabarranco.

Morales, F. (2014). “' 'Supervivientes’ regresa tres años después”. El País [en línea], 16 de marzo. Disponible en: http://cultura.elpais.com/cultura/2014/03/16/television/1395001120_525502.html [2014, 18 de marzo].

Moreno, P.S. (2005). "Ecotourism Along the Meso-American Caribbean Reef: The Impacts of Foreign Investment”. Human Ecology, 33 (2), 217-244.

Pérez Vanegas, M. et al. (2003). "Incidencia del uso de servicios integrados en el consumo de productos turísticos extrahoteleros”. Revista del Centro de Investigación, 6 (21), 89-97.

Pineda, E. y M. Aronne (2012). "Monitoreo de impactos turísticos en el Monumento Natural Marino Archipiélago Cayos Cochinos”. Inédito.

Secretaría de Relaciones Exteriores (2011). National Investment Promotion Program- Consolidated Index of Investment Projects. Tegucigalpa: Secretaría de Relaciones Exteriores.

Tourist Options (s. f.). "Cayos Cochinos, Honduras", en Tourist Options [en línea]. Disponible en: http://www.hondurastouristoptions.com/cayoscochinos.php\#cayos [2014, 6 de noviembre].

Young, K. et al. (2006). "Marine Field Research Summary. Cayos Cochinos Marine Site. June-September 2006” [en línea]. Fundación Cayos Cochinos/ Corporación Hondureña de Desarrollo Forestal. Disponible en: http:// opwall.com/wp-content/uploads/Cayos-Cochinos-Season-sciencesummary-2006.pdf [2014, 12 de diciembre]. 